\title{
Influencia de un recubrimiento comestible adicionado con calcio sobre la calidad de la mora de Castilla
}

\section{Influence of edible coating added with calcium on andean blackberry quality}

\author{
Misael Cortés-Rodríguez ${ }^{1}$; Rosa Alegría Macías²; Lina Cardona Velásquez ${ }^{3}$; Julio Cesar Arango Tobón ${ }^{4}$
}

${ }^{1}$ Ingeniero Químico, PhD. Ingeniería de Alimentos, Profesor Titular. Universidad Nacional de Colombia, Sede Medellín, Departamento de Ingeniería Agrícola y de Alimentos. Carrera 65 No. 59A - 110, Medellín, Colombia, e-mail: mcortesro@unal.edu.co, Dhttps://orcid.org/0000-0003-3407-1635

${ }^{2}$ Química, Esp. Biotecnología, MSc. Ciencia y Tecnología de Alimentos. Universidad Nacional de Colombia, Sede Medellín, Departamento de Ingeniería Agrícola y de Alimentos. Carrera 65 No. 59A - 110, Medellín, Colombia, e-mail: roaalegriama@unal.edu.co, Dhttps://orcid.org/0000-0002-2092-568X

${ }^{3}$ Ingeniera Biológica, MSc. Ciencia y Tecnología de Alimentos. Universidad Nacional de Colombia, Sede Medellín, Departamento de Ingeniería Agrícola y de Alimentos. Carrera 65 No. 59A - 110, Medellín, Colombia, e-mail: Imcardonav@unal.edu.co, Dhttps://orcid.org/0000-0003-2894-7063

${ }^{4}$ Ingeniero Agrícola, MSc. Aprovechamiento de Recursos Hidráulicos, Profesor Titular. Universidad Nacional de Colombia, Sede Medellín, Departamento de Ingeniería Agrícola y de Alimentos. Carrera 65 No. 59A - 110, Medellín, Colombia, e-mail: jcarango@unal.edu.co, Dhttps://orcid.org/0000-0003-1625-0541

Cómo citar: Cortés-Rodríguez, M.; Macías, R.A.; Cardona Velásquez, L.; Arango Tobón, J.C. 2019. influencia de un recubrimiento comestible adicionado con calcio sobre la calidad de la mora de Castilla. Rev. U.D.C.A Act. \& Div. Cient. 22(1):e1212. https://doi. org/10.31910/rudca.v22.n1.2019.1212

Artículo de acceso abierto publicado por Revista U.D.C.A Actualidad \& Divulgación Científica bajo una licencia Creative Commons CC BY-NC 4.0

Recibido: Junio 18 de 2018

Aceptado: Mayo 6 de 2019

\section{RESUMEN}

La creciente necesidad de los consumidores por alimentos saludables ha suscitado, en la industria, la búsqueda de tecnologías efectivas, que ofrezcan alimentos seguros y aceptables, sensorialmente, por el consumidor moderno. Los recubrimientos comestibles adicionados con componentes activos aplicados en estructuras vegetales, además de proteger la estructura y conferir mayor conservación, pueden aportar valor nutricional. El objetivo de la investigación fue evaluar la influencia de un recubrimiento comestible a base de alginato de sodio y calcio sobre los atributos de calidad de la mora de Castilla. Se aplicó la metodología de superficie de respuesta, con un diseño central compuesto cara centrado (21 experimentos), considerando las variables independientes: alginato de sodio (2,0$3,0 \%)$, cera de abeja $(0,5-1,0 \%)$, sucroéster $(0,15-0,25 \%)$, alginato/ glicerol $(2,0-3,0)$. La mejor formulación fue: alginato de sodio
$(2,85 \%)$, cera de abeja $(1,00 \%)$, alginato de sodio/glicerol $(2,0)$ y sucroéste $(0,18 \%)$, siendo los atributos de calidad de la mora con el recubrimiento comestible: humedad $(83,7 \pm 1,7 \%)$, actividad de agua $(0,964 \pm 0,010), \mathrm{pH}(2,7 \pm 0,0)$, acidez $(2,6 \pm 0,0 \%),{ }^{\circ} \operatorname{Brix}(8,1 \pm 0,2 \%)$, firmeza $(12,3 \pm 0,7 \mathrm{~N})$, fenoles totales $(105,3 \pm 4,5 \mathrm{mg} \mathrm{AG} / 100 \mathrm{~g})$, actividad antioxidante (ABTS: $962,7 \pm 15,1 \mathrm{mg}$ Trolox/100g y DPPH: $319,3 \pm 9,4 \mathrm{mg}$ Trolox/100g), luminosidad (22,5 $\pm 0,7)$; cromaticidad $a^{*}(8,47 \pm 0,88)$; cromaticidad $b^{*}(2,12 \pm 0,37)$ y calcio $=144,6 \pm 2,7$ $\mathrm{mg} / 200 \mathrm{~g}$. Los recubrimientos comestibles adicionados con calcio aplicados en la mora de Castilla representan una alternativa efectiva contra los daños mecánicos del fruto y para conservar su calidad, lo cual, podrá mejorar la vida útil durante el almacenamiento.

Palabras clave: Rubus glaucus; fortificación; optimización; conservación; calidad del alimento. 


\section{ABSTRACT}

The growing need of consumers for healthy food has led to the search for effective technologies in food industry that offer safe and sensorially acceptable options to the modern consumer. Edible coatings added with active components applied to vegetable structures, besides protecting the structure and conferring greater conservation, can provide nutritional value. The objective of this investigation was to evaluate the influence of an edible coating based on sodium alginate and calcium on the attributes of quality of Andean blackberry of Castilla. The response surface methodology was applied with a composite central design (21 experiments), considering the independent variables: sodium alginate (2.0-3.0\%), beeswax (0.5-1.0\%), sucroester (0.15-0.25\%), sodium alginate/ glycerol (2.0-3.0). The best formulation was: sodium alginate $(2.85 \%)$, beeswax $(1.00 \%)$, sodium alginate/glycerol $(2.0 \%)$ and sucroester $(0.162 \%)$, being the quality attributes of the of the blackberry with the edible coating: moisture $(83.7 \pm 1.7 \%)$, water activity $(0.964 \pm 0.010), \mathrm{pH}(2.7 \pm 0.0)$, acidity $(2.6 \pm 0.0 \%)$, ${ }^{\circ}$ Brix $(8.1 \pm 0.2 \%)$, firmness $(12.3 \pm 0.7 \mathrm{~N})$; total phenols $(105.3 \pm 4.5 \mathrm{mg}$ GA/100 g), antioxidant activity (ABTS: $962.7 \pm 15.1 \mathrm{mg}$ Trolox/100 g) and DPPH: $319.3 \pm 9.4 \mathrm{mg}$ Trolox/100 g), lightness (22,5 $\pm 0,7)$; chromaticity $\mathrm{a}^{*}(8,47 \pm 0,88)$; chromaticity $\mathrm{b}^{*}(2,12 \pm 0,37)$ and calcium $=144,6 \pm 2,7 \mathrm{mg} / 200 \mathrm{~g}$. Edible coatings added with calcium applied to Andean blackberry of Castilla represent an effective alternative against mechanical damages of the fruit and to conserve its quality, which will improve shelf life.

Keywords: Rubus glaucus; fortification; optimization; conservation; food quality.

\section{INTRODUCCIÓN}

La mora de Castilla (Rubus glaucus Benth) es una fruta rica en compuestos antioxidantes, como los polifenoles, que actúan como agentes secuestradores de radicales libres, brindando grandes beneficios a la salud, ya que logran reducir el riesgo de cáncer y enfermedades cardiovasculares (Oszmiański et al. 2015). La mora, debido a su valor nutricional, sabor y propiedades antioxidantes, es utilizada en la industria de alimentos, como materia prima para mermeladas, salsas, refrescos y dulces (Grijalba et al. 2010); además, aproximadamente el $90 \%$ de la producción internacional de mora, es transformada en productos procesados, como néctares, jugos, pulpas congeladas, concentrados, gelatinas y polvos deshidratados (Carvalho \& Betancour, 2015).

Sin embargo, este fruto presenta problemas en la conservación postcosecha, donde las mermas son del 25 al 50\%, ya que la mora posee una estructura morfológica frágil, lo que la hace susceptible a daños físicos, generación de lixiviados, pérdida de coloración, fermentación y proliferación de hongos (Ramírez et al. 2013). Estas pérdidas postcosecha afectan la economía de los productores y comerciantes: 15\% (países desarrollados) y 45\% (países en vía de desarrollo), reduciendo la competitividad de la cadena de producción (Rodríguez et al. 2010).
Bajo este contexto, se hace necesario la aplicación de tecnologías mejoradas, que disminuyan su deterioro y extiendan la vida útil. Se destacan, durante los últimos tiempos, algunas tecnologías aplicadas en frutos, tales como la irradiación UV, refrigeración, ozonización, atmósfera modificada y los recubrimientos comestibles (Falguera et al. 2011).

La tecnología de recubrimientos comestibles (RC) es amigable con el medio ambiente, aplicada en los alimentos por inmersión o aspersión, fijándose como una capa delgada y continua, que al secarse, proporciona una barrera contra $\mathrm{H}_{2} \mathrm{O}, \mathrm{O}_{2}$ y $\mathrm{CO}_{2}$, disminuye la velocidad de las reacciones químicas que provocan deterioro al alimento, permite un aporte nutricional al alimento y conserva mejor el alimento a temperaturas de refrigeración (Galus \& Kadzińska, 2015). Todos los beneficios del RC contribuyen a extender la vida útil del alimento, siendo su uso una tendencia en los productos mínimamente procesados (Galus \& Kadzińska, 2015; Kumari et al. 2017). Usualmente, el RC está conformado por proteínas animal o vegetal (proteínas lácteas, de gluten de trigo, soya), lípidos o ceras naturales (cera de abejas (CA), carnauba, candelilla, entre otros) que brindan brillo a los frutos recubiertos (Acevedo \& Soliva, 2017) y polisacáridos (alginatos, pectinas, carrageninas o mezclas, entre otros). Los lípidos, se incorporan en soluciones filmógenas para formar la estructura base de la emulsión del RC, siendo importante la estabilidad, la homogeneidad y la cohesividad del RC (Acevedo \& Soliva, 2017).

El objetivo de la investigación fue evaluar la influencia de un $\mathrm{RC}$ a base de alginato de sodio y calcio $(\mathrm{RC}+\mathrm{Ca})$, sobre los atributos de calidad de la mora de Castilla.

\section{MATERIALES Y MÉTODOS}

La mora de Castilla fue cosechada en el municipio de GranadaAntioquia, con un grado de madurez 5 y clasificada como "categoría extra”, según la Norma Técnica Colombiana (ICONTEC, 1997).

Se prepararon $21 \mathrm{RC}+$ Ca utilizando la metodología de superficie de respuesta, con un diseño central compuesto cara centrado (Tabla 1), siendo las variables independientes: alginato de sodio (AS) (2-3\%), CA $(0,5-1 \%)$, sucroéster (S) $(0,15-0,25 \%)$ y alginato de sodio/glicerol (AS/G) (2 -3) y las variables dependientes: humedad (Xw), actividad de agua (aw), ${ }^{\circ}$ Brix, $\mathrm{pH}$, acidez, fenoles totales (FT), capacidad antioxidante (ABTS y DPPH), textura y color.

Se utilizó quitosano (Q) (Sigma) (0,5\%p/p), como agente microbiano, disuelto en ácido acético $0,25 \mathrm{M}(1: 100)$, calentando hasta $40^{\circ} \mathrm{C}$ y homogenizando a 7000rpm (Ultra'Turrax IKA, T25), durante 10 min (premezcla $\mathrm{M}_{1}$ ). El AS, se disolvió en el $60 \%$ del agua de la formulación, calentando con agitación a $60^{\circ} \mathrm{C}$ hasta su completa hidratación y, finalmente, se adicionó el $\mathrm{G}$ (premezcla $\mathrm{M}_{2}$ ). Una premezcla $\mathrm{M}_{3}$, se preparó a partir de CA, 20\% del agua y el S; inicialmente, se mezcló la CA con agua caliente a $65^{\circ} \mathrm{C}$ y cuando se fundió la CA, se agregó el S. Posteriormente, a la premezcla M3, se le adicionó la premezcla $\mathrm{M}_{1}$ manteniendo la homogenización 
(7000rpm) y calentamiento constante a $65^{\circ} \mathrm{C}$, durante 2 min, obteniéndose la premezcla $\mathrm{M}_{4}$.

Una premezcla $\mathrm{M}_{5}$, se preparó con el $\mathrm{CaCl}_{2}(1,396 \%$ ) y el $20 \%$ de agua restante. La concentración de $\mathrm{CaCl}_{2}$ se determinó por un balance de materia, considerando el calcio en la mora fresca $(\approx 252,7 \mathrm{mg}$ calcio $/ \mathrm{kg})$, la cantidad de RC adherido/100g mora $(\approx 19 \mathrm{~g}$ RC) y fijando un objetivo teórico $(100 \mathrm{mg}$ calcio/119g mora $+\mathrm{RC}$ ). Después, se adicionó lentamente la premezcla $\mathrm{M}_{2}$ a la premezcla $\mathrm{M}_{4}$, obteniendo la premezcla Mo, la cual, se homogenizó a $15000 \mathrm{rpm}$ durante 5 min y manteniendo la temperatura de $65^{\circ} \mathrm{C}$ y así evitar que la CA solidifique. Al terminar de preparar la premezcla $\mathrm{M}_{6}$, se adicionó lentamente la premezcla $\mathrm{M}_{5}$, continuando la homogenización a 15000rpm, por 4 min más y 1 min a 20000rpm; finalmente, el $\mathrm{RC}+\mathrm{Ca}$, se desgasificó por $5 \mathrm{~min}$. Para su aplicación, los frutos fueron inicialmente lavados y desinfectados en una solución de $\mathrm{NaClO}$ a 50 ppm e inmersos en el $\mathrm{RC}+\mathrm{Ca}$, durante 1 min; posteriormente, se dejaron escurriendo durante $30 \mathrm{~s}$ y se colocaron en un secador de túnel (Centricol, Serie 0803) a $30^{\circ} \mathrm{C}$, 1600rpm, por $1 \mathrm{~h}$.

La caracterización de los atributos de calidad se realizó como sigue: Xw por el método A.O.A.C. 930.15/2012; la aw, AOAC 978.18/2012, con un higrómetro de punto de rocío a $25^{\circ} \mathrm{C}$ (Aqualab Decagón serie 3TE); ${ }^{\circ}$ Brix, pH y acidez, según la Norma Técnica Colombiana, NTC 4106 (ICONTEC, 1997). La extracción metanólica para la actividad antioxidante, se realizó por la metodología de Rodríguez et al. (2010) con modificaciones: se pesaron 0,3g de pulpa en tubos falcón de $50 \mathrm{~mL}$, se adicionaron $9 \mathrm{~mL}$ de una mezcla metanol/agua $70: 30$ y se centrifugó a $8000 \mathrm{rpm}$ por $10 \mathrm{~min}$, a $20^{\circ} \mathrm{C}$; posteriormente, el extracto se filtró usando papel Double Ring Qualitative Grade: medium 102. Los FT, se determinaron por el método colorimétrico Folin-Ciocalteu a 760nm, expresándose como mg ácido gálico/100 $\mathrm{g}$ muestra (mg AG/100 g). La actividad antioxidante sobre el radical DPPH (2,2-diphenyl-1-picrylhidrazyl), se determinó a $517 \mathrm{~nm}$ y sobre el radical $\mathrm{ABTS}^{+}$(ácido 2,2'-azino-bis(3-etilbenzotiazolin)-6sulfónico), a $734 \mathrm{~nm}$, de acuerdo con la metodología de Osmianski et al. (2015), expresándose como mg Trólox/100g.

El color, se determinó a partir de las coordenadas CIE-L*a*b*, utilizando un espectrofotómetro de esfera X-Rite, modelo SP64, iluminante D65, observador de $10^{\circ}$ y ventana de observación de 4 $\mathrm{mm}$. La textura se midió en términos de fuerza de ruptura $(\mathrm{Fr})$, según la metodología de Mannozzi et al. (2017), utilizando un texturómetro TA.XT2 (Stable Micro System), sonda P/5, velocidad y longitud de penetración de $2 \mathrm{~mm} / \mathrm{s}$ y $10 \mathrm{~mm}$, respectivamente. El análisis estadístico, se realizó con el software Statgraphics Centurion XVI. II, donde el ANOVA se realizó con un nivel de confianza del 95\% $(\mathrm{p} \leq 0,05)$.

\section{RESULTADOS Y DISCUSIÓN}

La calidad de la mora de Castilla fresca fue: $\mathrm{Xw}=85,6 \pm 1,2 \%$; $\mathrm{a}_{\mathrm{w}}=0,969 \pm 0,002 ; \mathrm{pH}=2,7 \pm 0,1$; acidez $=2,7 \pm 0,3 \%$; ${ }^{\circ}$ Brix $=$ $7,1 \pm 0,5 \%$; FT $=93,5 \pm 14,5 \mathrm{mg}$ AG/100g; DPPH = 299,9 $\pm 12,3 \mathrm{mg}$

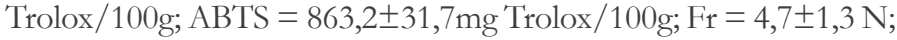

color $\left(L^{*}=23,6 \pm 0,5 ; a^{*}=21,4 \pm 6,0 y^{*}=7,1 \pm 2,5\right)$. Los resultados de $\mathrm{Xw}, \mathrm{aw}$, acidez, $\mathrm{pH}$ y ${ }^{\circ}$ Brix están relacionados con la estabilidad general del fruto, confiriendo un carácter perecedero, propenso al ataque microbiológico y corta vida útil (Ma et al. 2017).

Algunas investigaciones reportan, para la mora de Castilla, valores de Xw: 91,9; 87,7 y 82,0\% y aw: 0,981 y 0,984 (Franceschinis et al. 2014; Rodríguez et al. 2012). Otros autores reportan pH: 2,7 y 2,8; acidez: 1,65 y 1,67 (\%ácido cítrico) y ${ }^{\circ}$ Brix: 8,5 y 8,9 (Grijalba et al. 2010; Moreno et al. 2016) la calidad de la fruta cose- chada y la duración de las etapas de desarrollo de la flor y el fruto de dos materiales de mora de Castilla, con y sin espinas, cultivados en condiciones de campo abierto en el municipio de Cajicá (Cundinamarca. La variación de estas características depende de su madurez, de las condiciones freáticas del cultivo y de las prácticas agrícolas (Carvalho \& Betancour, 2015). Los FT y la actividad antioxidante obtenidos fueron altos en comparación con otros frutos (Oszmiański et al. 2015), lo que caracteriza a la mora de Castilla como un alimento funcional. En cuanto a la textura, Moreno et al. (2016) reportaron Fr entre 2,25 y 7,74 N, lo que confirma que este fruto presente daños mecánicos que contribuye a las importantes pérdidas postcosecha.

La tabla 1 presenta los atributos de calidad de la mora con $\mathrm{RC}+\mathrm{Ca}$ y la tabla 2 relaciona el ANOVA de las variables dependientes en función del valor $\mathrm{p}$, identificando sus diferencias significativas. Por otro lado, la figura 1 presenta los gráficos de superficie de respuesta de las variables dependientes, que fueron estadísticamente significativas.

La Xw de la mora con $\mathrm{RC}+\mathrm{Ca}(83,4-89,1 \%)$ fue similar a la fresca, lo que sugiere que esta depende principalmente, del proceso de secado aplicado y no por el aporte individual de agua de los componentes del $\mathrm{RC}+\mathrm{Ca}$. La aw presentó una situación similar a la $\mathrm{Xw}$, dado que el objetivo del secado es su reducción, la cual, depende del agua y del efecto depresor de los componentes presentes del $\mathrm{RC}+\mathrm{Ca}$. Igualmente, se observan valores similares de la aw en el fruto fresco $(0,969 \pm 0,002)$ y la mora con $\mathrm{RC}+\mathrm{Ca}(0,950-0,973)$. Los RC controlan la transferencia de agua desde el alimento al entorno, mejoran el sabor y olor propio del alimento durante el almacenamiento; además, ejercen mayor resistencia a los daños mecánicos de la misma, actuando como una barrera protectora (Zhang et al. 2014; Ali et al. 2012).

$\mathrm{El} \mathrm{pH}$ de las moras frescas y con $\mathrm{RC}+\mathrm{Ca}$ fueron similares, siendo los cambios de este último, poco significante: 2,66-2,83. Villegas \& Albarracín (2016) reportaron valores similares de $\mathrm{pH}(2,8)$ en mora con $\mathrm{RC}+$ hidroxypropilmetilcelulosa. La superficie de respuesta del $\mathrm{pH}$ (Figura 1) muestra que, a niveles altos de CA, el pH incrementa con el incremento de AS/G; mientras que, a niveles bajos de CA, el comportamiento es contrario. Esta situación podría atribuirse a los ácidos libres, ésteres y componentes naturales de la CA que, además aportan propiedades emulsificantes a la emulsión (Tosne et al. 2014) y al ácido acético presente en la formulación. El pH es importante desde el punto de vista sensorial en las frutas con y sin RC; cuando es alto, la percepción de dulzor incrementa, coherente con los cambios del $\mathrm{pH}$, reportados en fresas, por efecto de un $\mathrm{RC}$ 
Tabla 1. Análisis fisicoquímicos de mora de Castilla con RC+Ca.

\begin{tabular}{|c|c|c|c|c|c|c|c|c|c|c|c|c|c|c|c|c|}
\hline \multirow{2}{*}{ Exp } & \multirow{2}{*}{$\begin{array}{l}\text { AS } \\
(\%)\end{array}$} & \multirow{2}{*}{ AS/G } & \multirow{2}{*}{$\begin{array}{l}\text { CA } \\
(\%)\end{array}$} & \multirow{2}{*}{$\begin{array}{c}\mathrm{S} \\
(\%)\end{array}$} & \multirow{2}{*}{$\begin{array}{l}X_{w} \\
(\%)\end{array}$} & \multirow{2}{*}{$a_{w}$} & \multirow{2}{*}{$\mathrm{pH}$} & \multirow{2}{*}{$\begin{array}{c}\text { Acidez } \\
(\%)\end{array}$} & \multirow{2}{*}{${ }^{\circ}$ Brix } & \multirow{2}{*}{$\begin{array}{c}\text { FT } \\
\text { mg } \\
\mathrm{AG} / 100 \mathrm{~g}\end{array}$} & ABTS & \multirow{2}{*}{ DPPH } & \multicolumn{3}{|c|}{ Color } & \multirow{2}{*}{$\begin{array}{l}\text { Tex- } \\
\text { tura } \\
\text { (N) }\end{array}$} \\
\hline & & & & & & & & & & & $\mathrm{mg}$ Trolox/100g & & $\mathrm{a}^{*}$ & $\mathrm{~b}^{*}$ & $\mathrm{~L}^{*}$ & \\
\hline 1 & 2,00 & 2,00 & 0,50 & 0,15 & $\begin{array}{c}85,1 \pm \\
0,7\end{array}$ & $\begin{array}{c}0,960 \pm \\
0,009\end{array}$ & $\begin{array}{c}2,78 \pm \\
0,02\end{array}$ & $\begin{array}{c}2,2 \pm \\
0,1\end{array}$ & $\begin{array}{c}7,8 \pm \\
0,2\end{array}$ & $\begin{array}{c}100,3 \pm \\
7,6\end{array}$ & $\begin{array}{c}1044,4 \pm \\
11,9\end{array}$ & $\begin{array}{c}238,2 \pm \\
3,4\end{array}$ & $\begin{array}{c}21,6 \pm \\
1,9\end{array}$ & $\begin{array}{c}6,9 \pm \\
0,6\end{array}$ & $\begin{array}{c}22,1 \pm \\
1,5\end{array}$ & $\begin{array}{c}10,4 \pm \\
0,7\end{array}$ \\
\hline 3 & 2,50 & 2,50 & 0,75 & 0,25 & $\begin{array}{c}87,2 \pm \\
0,9\end{array}$ & $\begin{array}{c}0,973 \pm \\
0,002\end{array}$ & $\begin{array}{c}2,74 \pm \\
0,03\end{array}$ & $\begin{array}{c}2,3 \pm \\
0,3\end{array}$ & $\begin{array}{c}7,4 \pm \\
0,1\end{array}$ & $\begin{array}{c}92,4 \pm \\
10,4\end{array}$ & $\begin{array}{c}863,1 \pm \\
62,5\end{array}$ & $\begin{array}{c}346,5 \pm \\
4,3\end{array}$ & $\begin{array}{c}24,6 \pm \\
3,7\end{array}$ & $\begin{array}{c}8,9 \pm \\
2,1\end{array}$ & $\begin{array}{c}25,1 \pm \\
1,1\end{array}$ & $9,6 \pm 0,3$ \\
\hline 4 & 2,50 & 2,50 & 0,75 & 0,20 & $\begin{array}{c}89,1 \pm \\
1,9\end{array}$ & $\begin{array}{c}0,968 \pm \\
0,001\end{array}$ & $\begin{array}{c}2,72 \pm \\
0,01\end{array}$ & $\begin{array}{c}2,8 \pm \\
0,2\end{array}$ & $\begin{array}{c}7,6 \pm \\
0,2\end{array}$ & $\begin{array}{c}113,8 \pm \\
6,1\end{array}$ & $\begin{array}{c}923,1 \pm \\
48,4\end{array}$ & $\begin{array}{c}250,0 \pm \\
18,3\end{array}$ & $\begin{array}{c}24,9 \pm \\
4,2\end{array}$ & $\begin{array}{c}9,8 \pm \\
3,1\end{array}$ & $\begin{array}{c}27,9 \pm \\
4,4\end{array}$ & $\begin{array}{c}14,3 \pm \\
0,6\end{array}$ \\
\hline 7 & 2,00 & 2,00 & 1,00 & 0,15 & $\begin{array}{c}84,5 \pm \\
0,6\end{array}$ & $\begin{array}{c}0,965 \pm \\
0,002\end{array}$ & $\begin{array}{c}2,73 \pm \\
0,01\end{array}$ & $\begin{array}{c}3,2 \pm \\
0,3\end{array}$ & $\begin{array}{c}7,3 \pm \\
0,0\end{array}$ & $\begin{array}{c}138,5 \pm \\
4,5\end{array}$ & $\begin{array}{c}761,7 \pm \\
11,2\end{array}$ & $\begin{array}{c}297,6 \pm \\
4,5\end{array}$ & $\begin{array}{c}18,1 \pm \\
1,4\end{array}$ & $\begin{array}{c}5,3 \pm \\
0,4\end{array}$ & $\begin{array}{c}21,6 \pm \\
1,3\end{array}$ & $\begin{array}{c}11,6 \pm \\
0,7\end{array}$ \\
\hline 8 & 3,00 & 2,50 & 0,75 & 0,20 & $\begin{array}{c}85,1 \pm \\
1,5\end{array}$ & $\begin{array}{c}0,954 \pm \\
0,002\end{array}$ & $\begin{array}{c}2,80 \pm \\
0,02\end{array}$ & $\begin{array}{c}2,5 \pm \\
0,3\end{array}$ & $\begin{array}{c}7,9 \pm \\
0,1\end{array}$ & $\begin{array}{c}106,4 \pm \\
13,0\end{array}$ & $\begin{array}{c}574,9 \pm \\
14,4\end{array}$ & $\begin{array}{c}333,8 \pm \\
7,0\end{array}$ & $\begin{array}{c}24,8 \pm \\
1,0\end{array}$ & $\begin{array}{c}8,7 \pm \\
, 7\end{array}$ & $\begin{array}{c}21,4 \pm \\
0,9\end{array}$ & $\begin{array}{c}12,3 \pm \\
0,1\end{array}$ \\
\hline 9 & 2,00 & 3,00 & 0,50 & 0,25 & $\begin{array}{c}85,5 \pm \\
1,5\end{array}$ & $\begin{array}{c}0,958 \pm \\
0,00\end{array}$ & $\begin{array}{c}2,78 \pm \\
0,01\end{array}$ & $\begin{array}{c}2,3 \pm \\
0,1\end{array}$ & $\begin{array}{c}7,6 \pm \\
0,1\end{array}$ & $\begin{array}{c}90,7 \pm \\
13,0\end{array}$ & $\begin{array}{c}808,4 \pm \\
60,1\end{array}$ & $\begin{array}{c}325,7 \pm \\
5,6\end{array}$ & $\begin{array}{c}16,1 \pm \\
3,2\end{array}$ & $\begin{array}{c}8,2 \pm \\
5,0\end{array}$ & $\begin{array}{c}21,9 \pm \\
4,6\end{array}$ & $\begin{array}{c}11,1 \pm \\
0,6\end{array}$ \\
\hline 10 & 2,50 & 2,50 & 1,00 & 0,20 & $\begin{array}{c}86,2 \pm \\
1,3\end{array}$ & $\begin{array}{c}0,956 \pm \\
0,001\end{array}$ & $\begin{array}{c}2,83 \pm \\
0,01\end{array}$ & $\begin{array}{c}2,5 \pm \\
0,2\end{array}$ & $\begin{array}{c}7,7 \pm \\
0,1\end{array}$ & $\begin{array}{c}104,5 \pm \\
11,4\end{array}$ & $\begin{array}{c}554,4 \pm \\
13,1\end{array}$ & $\begin{array}{c}306,1 \pm \\
8,2\end{array}$ & $\begin{array}{c}19,8 \pm \\
6,8\end{array}$ & $\begin{array}{c}7,3 \pm \\
3,8\end{array}$ & $\begin{array}{c}21,1 \pm \\
1,3\end{array}$ & $\begin{array}{c}10,8 \pm \\
0,4\end{array}$ \\
\hline 14 & 3,00 & 2,00 & 0,50 & 0,25 & $\begin{array}{c}86,3 \pm \\
1,2\end{array}$ & $\begin{array}{c}0,957 \pm \\
0,00\end{array}$ & $\begin{array}{c}2,70 \pm \\
0,01\end{array}$ & $2,6 \pm 0,3$ & $\begin{array}{c}7,5 \pm \\
0,1\end{array}$ & $\begin{array}{c}110,0 \pm \\
7,0\end{array}$ & $\begin{array}{c}1136,2 \pm \\
14,9\end{array}$ & $\begin{array}{c}322,5 \pm \\
11,9\end{array}$ & $\begin{array}{c}24,4 \pm \\
3,1\end{array}$ & $\begin{array}{c}7,6 \pm \\
1,3\end{array}$ & $\begin{array}{c}28,5 \pm \\
0,6\end{array}$ & $8,8 \pm 0,3$ \\
\hline 15 & 2,00 & 2,50 & 0,75 & 0,20 & $\begin{array}{c}86,2 \pm \\
0,5\end{array}$ & $\begin{array}{c}0,955 \pm \\
0,00\end{array}$ & $\begin{array}{c}2,68 \pm \\
0,01\end{array}$ & $\begin{array}{c}2,7 \pm \\
0,2\end{array}$ & $\begin{array}{c}7,5 \pm \\
0,0\end{array}$ & $\begin{array}{c}106,7 \pm \\
3,9\end{array}$ & $\begin{array}{c}875,6 \pm \\
46,8\end{array}$ & $\begin{array}{c}270,6 \pm \\
9,9\end{array}$ & $\begin{array}{c}26,0 \pm \\
7,0\end{array}$ & $\begin{array}{c}8,5 \pm \\
3,4\end{array}$ & $\begin{array}{c}28,1 \pm \\
2,7\end{array}$ & $8,9 \pm 0,1$ \\
\hline 16 & 3,00 & 2,00 & 1,00 & 0,25 & $\begin{array}{c}85,5 \pm \\
0,9\end{array}$ & $\begin{array}{c}0,959 \pm \\
0,00\end{array}$ & $\begin{array}{c}2,71 \pm \\
0,01\end{array}$ & $\begin{array}{c}3,5 \pm \\
0,3\end{array}$ & $\begin{array}{c}7,6 \pm \\
0,0\end{array}$ & $\begin{array}{c}108,5 \pm \\
9,6\end{array}$ & $\begin{array}{c}915,5 \pm \\
25,3\end{array}$ & $\begin{array}{c}240,8 \pm \\
12,6\end{array}$ & $\begin{array}{c}17,9 \pm \\
3,5\end{array}$ & $\begin{array}{c}5,7 \pm \\
1,3\end{array}$ & $\begin{array}{c}21,6 \pm \\
1,0\end{array}$ & $\begin{array}{c}10,8 \pm \\
0,6\end{array}$ \\
\hline 17 & 2,50 & 2,50 & 0,75 & 0,20 & $\begin{array}{c}85,5 \pm \\
1,5\end{array}$ & $\begin{array}{c}0,963 \pm \\
0,002\end{array}$ & $\begin{array}{c}2,71 \pm \\
0,01\end{array}$ & $\begin{array}{c}3,1 \pm \\
0,6\end{array}$ & $\begin{array}{c}7,7 \pm \\
0,0\end{array}$ & $\begin{array}{c}99,3 \pm \\
12,0\end{array}$ & $\begin{array}{c}1026,7 \pm \\
26,2\end{array}$ & $\begin{array}{c}362,5 \pm \\
8,8\end{array}$ & $\begin{array}{c}19,2 \pm \\
5,7\end{array}$ & $\begin{array}{c}6,08 \pm \\
2,52\end{array}$ & $\begin{array}{c}22,9 \pm \\
0,6\end{array}$ & $9,6 \pm 0,2$ \\
\hline 18 & 2,50 & 2,50 & 0,50 & 0,20 & $\begin{array}{c}85,2 \pm \\
1,9\end{array}$ & $\begin{array}{c}0,967 \pm \\
0,001\end{array}$ & $\begin{array}{c}2,70 \pm \\
0,02\end{array}$ & $\begin{array}{c}2,2 \pm \\
0,2\end{array}$ & $\begin{array}{c}7,7 \pm \\
0,1\end{array}$ & $\begin{array}{c}113,0 \pm \\
11,9\end{array}$ & $\begin{array}{c}781,3 \pm \\
2,9\end{array}$ & $\begin{array}{c}384,1 \pm \\
10,5\end{array}$ & $\begin{array}{c}22,0 \pm \\
2,1\end{array}$ & $\begin{array}{c}7,06 \pm \\
1,08\end{array}$ & $\begin{array}{c}22,5 \pm \\
0,8\end{array}$ & $\begin{array}{c}10,3 \pm \\
0,2\end{array}$ \\
\hline 19 & 2,50 & 2,50 & 0,75 & 0,20 & $\begin{array}{c}85,2 \pm \\
1,3\end{array}$ & $\begin{array}{c}0,968 \pm \\
0,002\end{array}$ & $\begin{array}{c}2,80 \pm \\
0,03\end{array}$ & $\begin{array}{c}2,2 \pm \\
0,1\end{array}$ & $\begin{array}{c}8,3 \pm \\
0,2\end{array}$ & $\begin{array}{c}111,9 \pm \\
8,3\end{array}$ & $\begin{array}{c}701,4 \pm \\
23,6\end{array}$ & $\begin{array}{c}345,3 \pm \\
1,5\end{array}$ & $\begin{array}{c}19,0 \pm \\
3,2\end{array}$ & $\begin{array}{c}6,90 \pm \\
3,53\end{array}$ & $\begin{array}{c}24,38 \pm \\
0,9\end{array}$ & $9,6 \pm 0,4$ \\
\hline 20 & 3,00 & 3,00 & 0,50 & 0,15 & $\begin{array}{c}85,8 \pm \\
1,3\end{array}$ & $\begin{array}{c}0,966 \pm \\
0,001\end{array}$ & $\begin{array}{c}2,72 \pm \\
0,01\end{array}$ & $\begin{array}{c}2,4 \pm \\
0,2\end{array}$ & $\begin{array}{c}6,62 \pm \\
0,2\end{array}$ & $\begin{array}{c}102,8 \pm \\
3,9\end{array}$ & $\begin{array}{c}675,5 \pm \\
16,7\end{array}$ & $\begin{array}{c}338,5 \pm \\
8,0\end{array}$ & $\begin{array}{c}14,1 \pm \\
2,7\end{array}$ & $\begin{array}{c}4,53 \pm \\
1,53\end{array}$ & $\begin{array}{c}21,2 \pm \\
0,2\end{array}$ & $9,5 \pm 0,5$ \\
\hline 21 & 2,50 & 2,50 & 0,75 & 0,20 & $\begin{array}{c}85,6 \pm \\
1,3\end{array}$ & $\begin{array}{c}0,957 \pm \\
0,003\end{array}$ & $\begin{array}{c}2,70 \pm \\
0,01\end{array}$ & $\begin{array}{c}2,5 \pm \\
0,2\end{array}$ & $\begin{array}{c}6,6 \pm \\
0,1\end{array}$ & $\begin{array}{c}94,3 \pm \\
3,4\end{array}$ & $\begin{array}{c}628,2 \pm \\
16,8\end{array}$ & $\begin{array}{c}238,2 \pm \\
2,9\end{array}$ & $\begin{array}{c}22,4 \pm \\
4,2\end{array}$ & $\begin{array}{c}8,44 \pm \\
2,58\end{array}$ & $\begin{array}{c}24,8 \pm \\
2,8\end{array}$ & $\begin{array}{c}12,1 \pm \\
0,6\end{array}$ \\
\hline
\end{tabular}



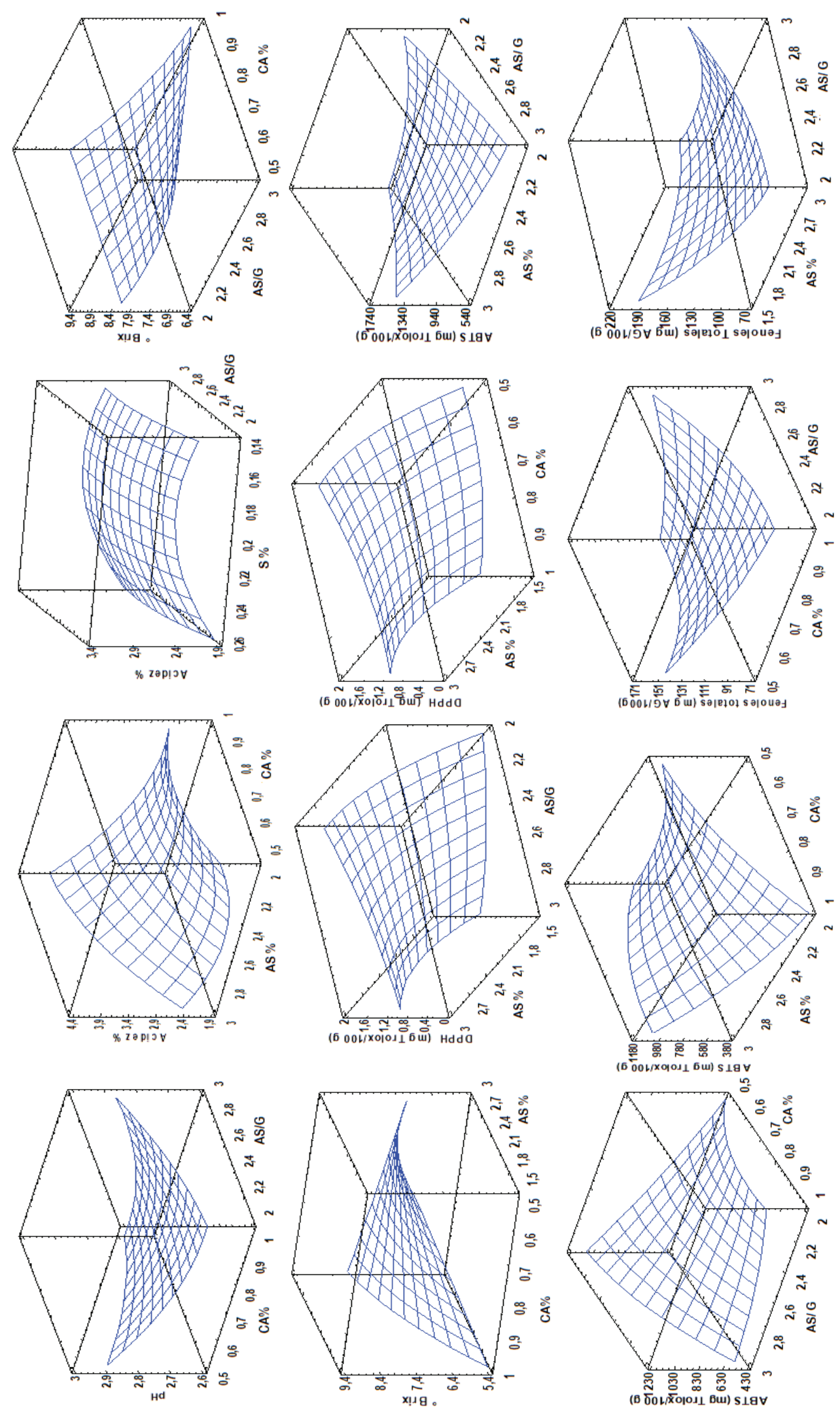

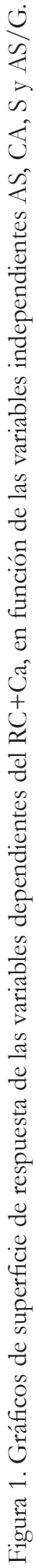



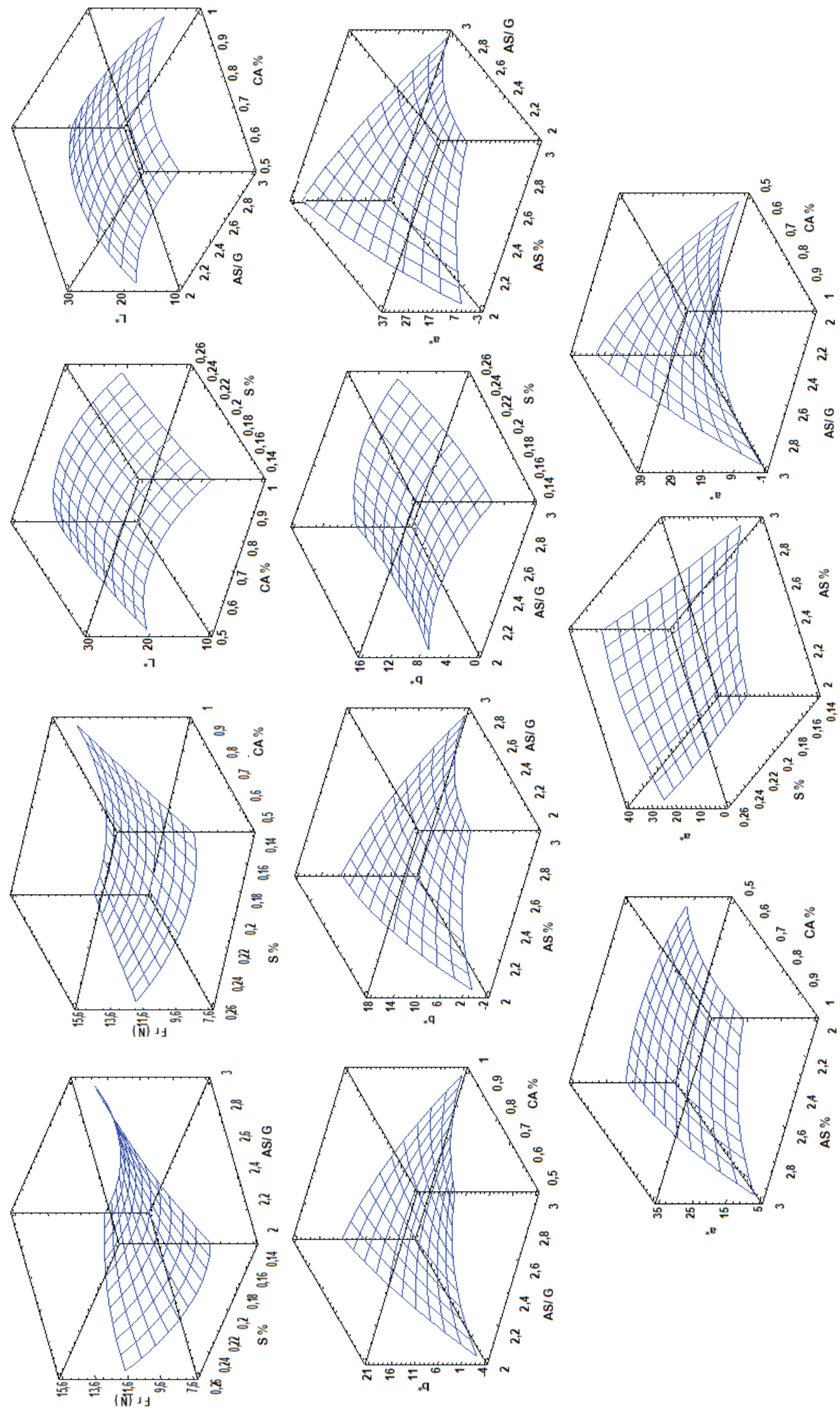

 
Tabla 2. ANOVA para la mora de Castilla con $\mathrm{RC}+\mathrm{Ca}$.

\begin{tabular}{|c|c|c|c|c|c|c|c|c|c|c|c|c|c|c|}
\hline & \multicolumn{4}{|c|}{ Efectos principales } & \multicolumn{4}{|c|}{ Efectos cuadráticos } & \multicolumn{6}{|c|}{ Efectos por interacciones } \\
\hline & $\mathbf{A}$ & B & $\mathrm{C}$ & $\mathbf{D}$ & AA & BB & $\mathrm{CC}$ & DD & $\mathrm{AB}$ & AC & $\mathrm{AD}$ & BC & BD & $\mathrm{CD}$ \\
\hline $\mathrm{pH}$ & 0,297 & $0,000^{*}$ & 0,923 & 0,255 & 0,136 & $0,025^{*}$ & $0,000^{*}$ & 0,956 & 0,198 & 0,799 & 0,471 & $0,000^{*}$ & $0,001 *$ & 0,112 \\
\hline Acidez & $0,000^{*}$ & $0,040^{*}$ & $0,000^{*}$ & $0,015^{*}$ & $0,000^{*}$ & 0,270 & $0,048^{*}$ & 0,056 & 0,002 & $0,008^{*}$ & 0,201 & 0,880 & 0,963 & 0,515 \\
\hline${ }^{\circ}$ Brix & 0,259 & $0,000^{*}$ & $0,020^{*}$ & 0,923 & 0,190 & $0,011 *$ & 0,894 & $0,001 *$ & 0,776 & $0,000^{*}$ & 0,633 & 0,492 & 0,039 & 0,813 \\
\hline $\mathbf{X w}$ & 0,370 & 0,257 & 0,756 & $0,037^{*}$ & 0,333 & $0,026^{*}$ & 0,317 & 0,086 & 0,313 & $0,048^{*}$ & 0,309 & 0,928 & 0,839 & 0,822 \\
\hline$a_{w}$ & 0,590 & $0,000 *$ & $0,021 *$ & 0,634 & $0,000 *$ & $0,926^{*}$ & $0,049 *$ & $0,000^{*}$ & $0,184^{*}$ & 0,057 & 0,402 & $0,062^{*}$ & $0,281 *$ & $0,036^{*}$ \\
\hline FT & 0,418 & 0,807 & 0,290 & $0,000^{*}$ & 0,086 & $0,001 *$ & 0,152 & 0,062 & $0,050^{*}$ & 0,838 & 0,191 & $0,008^{*}$ & 0,375 & 0,306 \\
\hline ABTS & $0,010^{*}$ & $0,000^{*}$ & $0,000^{*}$ & $0,000^{*}$ & $0,000^{*}$ & 0,117 & $0,000^{*}$ & $0,000^{*}$ & $0,000^{*}$ & $0,000^{*}$ & 0,416 & $0,000^{*}$ & $0,000^{*}$ & $0,000 *$ \\
\hline DPPH & 0,401 & $0,017^{*}$ & $0,000^{*}$ & $0,005^{*}$ & $0,000^{*}$ & $0,000^{*}$ & $0,000^{*}$ & 0,351 & $0,015^{*}$ & $0,000^{*}$ & 0,056 & $0,040^{*}$ & 0,340 & 0,070 \\
\hline $\mathrm{L}^{*}$ & 0,751 & 0,498 & $0,017^{*}$ & $0,016^{*}$ & 0,384 & $0,016^{*}$ & $0,000^{*}$ & 0,627 & 0,705 & 0,365 & 0,130 & 0,189 & 0,877 & 0,994 \\
\hline$a^{*}$ & $0,000^{*}$ & 0,318 & $0,024 *$ & $0,000^{*}$ & $0,028^{*}$ & $0,000^{*}$ & $0,011^{*}$ & 0,495 & $0,000^{*}$ & $0,001^{*}$ & $0,000^{*}$ & $0,000^{*}$ & 0,65 & 0,054 \\
\hline$b^{*}$ & 0,144 & 0,904 & 0,330 & $0,041 *$ & 0,099 & $0,008^{*}$ & 0,095 & 0,502 & 0,014* & 0,969 & 0,899 & $0,000^{*}$ & 0,402 & 0,721 \\
\hline Fr & 0,890 & 0,491 & 0,069 & 0,564 & 0,137 & 0,266 & 0,938 & 0,094 & 0,106 & 0,599 & 0,007 & 0,523 & $0,002 *$ & $0,002 *$ \\
\hline
\end{tabular}

Factor A: AS (\%), Factor B: AS/G, Factor C: CA (\%), Factor D: S (\%). *Significativo $(p<0,05)$.

a base de Q y CA (Velickova et al. 2013). Para la mora con RC+Ca, los mayores $\mathrm{pH}$ se obtienen a AS/G y CA de $(2,0 ; 0,5)$ y $(3,0 ; 1,0)$, respectivamente. La superficie de respuesta de la acidez (Figura 1), muestra la tendencia de incrementar a mayores contenidos de AS $(3 \%)$ y CA $(1 \%)$, lo cual es coherente con los cambios observados en el $\mathrm{pH}$ y atribuibles, principalmente, a la CA y al ácido acético de las formulaciones. El efecto del S y de la relación AS/G, se considera menos influyente sobre la acidez; hay una tendencia de incrementar la acidez con la disminución del $\mathrm{S}$ y con el incremento de la relación, pero a bajos niveles de $\mathrm{S}(0,14 \%)$.

Los valores medios de los ${ }^{\circ}$ Brix de la mora con $\mathrm{RC}+\mathrm{Ca}$ fluctuaron entre 6,57 y $8,65 \%$, siendo en algunas formulaciones mayores a la mora fresca $(7,1 \pm 0,5 \%)$; por lo que, se deduce que existen efectos de los componentes del $\mathrm{RC}+\mathrm{Ca}$. La superficie de respuesta CA-AS para los ${ }^{\circ}$ Brix (Figura 1) identifica una interacción negativa entre estos factores, alcanzando mayores ${ }^{\circ}$ Brix a altos niveles de AS (CA $=1 \%$ ) y a bajos niveles de CA (AS $=1,5 \%$ ), lo cual, se atribuye, principalmente, al aporte de sólidos solubles del AS al solubilizarse en la fase acuosa. Por otro lado, existe una tendencia a aumentar los ${ }^{\circ}$ Brix con la disminución de la relación AS/G, especialmente, a niveles de CA del 1\% y con la disminución de la CA, esencialmente, con relación $A S / G$ de 3 . Esta situación no es clara, ya que la disminución de la relación implica dos situaciones; por un lado, una menor contribución de sólidos solubles por parte del AS, lo cual, no favorecería el incremento de los ${ }^{\circ}$ Brix y, por otro lado, un incremento del $\mathrm{G}$ en la formulación, más coherente con los resultados obtenidos, ya que implicaría un mayor aporte de sólidos solubles de éste, dada la solubilidad en fase acuosa que tiene, por los grupos de hidróxidos $(\mathrm{OH})$ presentes. Otro posible factor que podría incrementar los ${ }^{\circ}$ Brix son las pérdidas de Xw de la mora con $\mathrm{RC}+\mathrm{Ca}$ durante el secado (Ramírez et al. 2013). Se considera que el efecto del $\mathrm{S}$ no es tan crítico, dado que los cambios observados están del orden de $0,4^{\circ}$ Brix, aproximadamente.
En general, es deseable que los ${ }^{\circ}$ Brix en las frutas sea alto (Grijalba et al. 2010) y, principalmente, en frutas ácidas, lo que mejora el balance dulzor -acidez. La mora de Castilla con estado de madurez 5 contiene entre 7,2 y 7,9 ${ }^{\circ}$ Brix (ICONTEC, 1997). Oliveira et al. (2014) reportaron en mora fresca $\left(7,5^{\circ} \mathrm{Brix}\right)$, mora con $\mathrm{RC}$ a base de $\mathrm{Q}\left(7,3^{\circ} \mathrm{Brix}\right)$ y mora con $\mathrm{RC}$ a base de almidón de tapioca al 2,5\%. (7,4 ${ }^{\circ}$ Brix). Por otro lado, la aplicación de RC a base de $\mathrm{Q}$ en mango troceado (Chien et al. 2007) y en fresas (Hernández-Muñoz et al. 2008), disminuyó la pérdida de Xw y aumentaron los ${ }^{\circ}$ Brix.

En cuanto a los FT y la capacidad antioxidante, los valores medios obtenidos con RC+Ca (FT: 86,0 a 141,4mg AG/100g; ABTS = 574,9 a 1136,0mg Trolox/100g y DPPH = 238,2 a 384,1 mg Trolox/100g), denotan diferencias significativas con respecto a los valores obtenidos

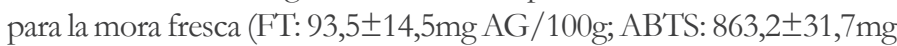
Trolox/100g y DPPH: 299,9 $\pm 12,3 \mathrm{mg}$ Trolox/100g). Estas diferencias, igualmente han sido reportadas por López-Mata et al. (2012) en fresas con un RC a base de almidón, aceite de canela y Q. Esta situación identifica una influencia de los componentes del $\mathrm{RC}+\mathrm{Ca}$ sobre la actividad antioxidante, donde se destaca, en principio, la CA (ABTS:

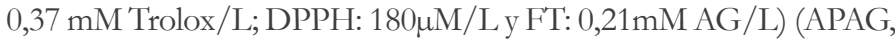
2016) y el Q (ABTS: 0,026mmol Trolox/100mL; DPPH: 35\% actividad antiradical) (García et al. 2014). La mora de Castilla es un fruto con una capacidad antioxidante relevante (Garzón et al. 2009), aportado por compuestos fenólicos, vitaminas, entre otros (LópezMata et al. 2012); sin embargo, la variabilidad observada podría estar siendo afectada por otros factores: estado de madurez, componentes del suelo, climatología, incidencia de la luz durante su crecimiento, entre otros (Rodríguez et al. 2010).

La superficie de respuesta de los FT (Figura 1) muestran que la mora con $\mathrm{RC}+\mathrm{Ca}$ se potencia, principalmente, a bajos niveles de CA, AS y AS/G; por lo que, no hay una tendencia muy definida de los cambios observados. Por otro lado, el ABTS y el 
DPPH presentaron mucha variabilidad, potenciándose el ABTS, especialmente, a bajos contenidos de CA y altos contenidos de AS y AS/G. Estos resultados podrían estar asociados a una influencia en la extracción metanólica, debido a la estructura molecular de los analitos presentes, que permiten que haya mayor proporción extraíble de estos compuestos con capacidad antioxidante. Otra causa de este aumento podría ser debido a la pérdida de agua que pudo experimentar la fruta, dando lugar a mayor concentración de los compuestos antioxidantes (Oliveira et al. 2014). Los compuestos fenólicos aportan una importante actividad antioxidante, asociada con alimentos de calidad, aceptabilidad y estabilidad. El contenido de FT reportado en esta investigación concuerda con los valores reportados por Ghasemnezhad et al. (2010) en albaricoque con RC a base de Q. Otros trabajos reportan que las frutas con RC+AS presentan una menor pérdida del contenido fenólico con respecto a la fruta fresca durante el almacenamiento, debido a la protección que ofrece el RC (Petriccione et al. 2015; Kerch et al. 2011; Kerch. 2015; Pérez-Gallardo et al. 2014).

En cuanto a la textura, se resalta que la mora con $\mathrm{RC}+\mathrm{Ca}$ presentó una mayor resistencia mecánica que la mora fresca $(\mathrm{Fr}=4,7 \pm 1,3$ $\mathrm{N}$ ), fluctuando sus valores medios entre 8,08 y $14,28 \mathrm{~N}$, lo cual, refleja, de manera importante, un mejoramiento contra los daños mecánicos del fruto. Esta situación denota un efecto importante de la relación AS-calcio, cuyas moléculas generan un sistema coloidal más complejo, dado que la emulsión se encontrará en un medio de baja fuerza de gel, debido a las altas relaciones $\mathrm{mg}$ calcio/g AS encontradas en las formulaciones $(361,3$ - 544,6) y ajustadas, según balances de materia, para alcanzar 100mg calcio /119g mora con $\mathrm{RC}+\mathrm{Ca}$. Bajo este contexto, la aplicación del $\mathrm{RC}+\mathrm{Ca}$ sobre la superficie de la mora y el secado posterior, potencia la resistencia mecánica por la mayor cohesión de sus moléculas, además de potenciar también su valor nutricional al fortificar la mora por la adición del calcio. Algunos autores han reportado que la adición de calcio en RC genera una mayor resistencia mecánica, además de ser estables estructuralmente, también inhiben la pérdida de firmeza en frutas frescas (Hernández-Muñoz et al. 2008).

La textura de la mora con $\mathrm{RC}+\mathrm{Ca}$ muestra que la resistencia mecánica del fruto se favorece a niveles bajos de $\mathrm{S}(0,14 \%)$ y altos niveles de CA (1\%) y AS/G (3\%), lo cual, puede ser atribuible a interacciones estructurales entre los componentes presentes del RC, que quedan atrapados en la red gelificada y seca AS-calcio que, a mayores contenidos de AS, reduce la relación mg calcio/g AS, favoreciendo una mayor fuerza de gel y mayor rigidez de la película seca adherida en la superficie del fruto. Por otro lado, la inclusión de plastificantes, como el $\mathrm{G}$ en la emulsión, mejora la flexibilidad y la funcionalidad de los RC, ayudando a mantener la firmeza de los frutos (RojasGraü et al. 2007). Se considera que el poder emulsificante del S, a concentraciones superiores, otorga una mayor estabilidad al sistema coloidal, confiriendo al fruto con $\mathrm{RC}+\mathrm{Ca}$ una película uniforme con mejores características de firmeza, mejorando notablemente su textura.

Los valores medios del color fluctuaron para L* $(16,3-28,5), a^{*}$ $(16,0-24,9)$ y $b^{*}(4,5-14,5)$. Estos resultados denotan diferencias con respecto a la mora fresca $\left(L^{*}=23,6 \pm 0,5 ; a^{*}=21,4 \pm 6,0 \mathrm{y} b^{*}\right.$
$=7,12 \pm 2,5)$, por lo que el $\mathrm{RC}+\mathrm{Ca}$ confiere una percepción del color diferente que depende de la formulación del RC+Ca utilizada. Las superficies de respuesta de los parámetros de color (Figura 1) presentan curvaturas, debido a las interacciones cuadráticas, observándose mayores cambios en L*, cuando existe un predominio de las interacciones en general y no de manera individual; para la mora, una percepción de mejor color se potencia a menores valores de $\mathrm{L}^{*}$, para minimizar la blancura observada en la emulsión del RC+Ca; al mismo tiempo, la intensidad o saturación del fruto se favorece con la maximización de $\mathrm{a}^{*}$ y la minimización de $\mathrm{b}^{*}$, confiriendo una tonalidad o matiz morado, acorde al fruto. En este contexto, las mejores formulaciones para $\mathrm{b}^{*}$ se dan con las interacciones CA-AS/G de $(1 \%, 3)$ y $(0,5 \%, 2)$, mientras que $a^{*}$, se da, principalmente, con la interacción de AS/G con CA, en (3, 0,5\%) y con el AS en (3, 2\%). Una de las posibles causas de los cambios de color observados podría ser atribuido al proceso de secado, debido a la modificación del índice de refracción de la superficie seca y porosa, que hace que la adsorción de luz sea menor e incremente L* cuando está más seca (Franceschinis et al. 2014). Algunos autores han reportado la conservación del color de los frutos durante el almacenamiento, utilizando RC+AS: Raybaudi-Massilia et al. (2008), en melón y Mantilla et al. (2012), en piña.

Optimización. La tabla 3 presenta los criterios fijados para cada variable dependiente en la optimización experimental de múltiples respuestas para la formulación del $\mathrm{RC}+\mathrm{Ca}$, donde se priorizó el peso e impacto de la Fr (>resistencia mecánica del fruto). Los resultados obtenidos fueron los siguientes: AS (2,85\%), CA (1,0\%), AS/G $(2,00)$ y S $(0,18 \%)$, con un valor de deseabilidad de 0,73 . Por otro lado, se presentan los valores predichos por los modelos y los valores experimentales, obtenidos a partir de 3 réplicas realizadas a las condiciones óptimas, los cuales, presentaron, en la mayoría, un error residual bajo y aceptable (excepto color, ABTS y FT), lo que, valida, estos modelos, utilizando la función de deseabilidad. Se resalta un contenido de calcio equivalente a $144,6 \pm 2,7 \mathrm{mg}$ calcio/200g de mora de Castilla, con $\mathrm{RC}+\mathrm{Ca}(\approx 14,5 \pm 0,3 \% \mathrm{VDR} / 200 \mathrm{~g})$.

El RC+Ca aplicado permitió obtener valores bajos de humedad y aw, mientras que, en otros parámetros, como actividad antioxidante, Fr y ${ }^{\circ}$ Brix, se presentó aumento en los valores obtenidos. Este comportamiento es concordante con lo propuesto en la tabla 3. El $\mathrm{RC}+\mathrm{Ca}$ aplicado en mora de Castilla tuvo un efecto positivo sobre la textura, la capacidad antioxidante y los FT; además, permitió obtener valores de $\mathrm{pH},{ }^{\circ}$ Brix y acidez, que cumplen con la norma técnica colombiana. Esta situación representa una alternativa eficaz para proteger la mora de Castilla contra los daños físicos que experimenta la fruta durante la etapa de postcosecha; además, para conservar su calidad, lo cual, podrá mejorar la vida útil durante el almacenamiento. Por otro lado, los $\mathrm{RC}+\mathrm{Ca}$ se presentan como un vehículo para la fortificación con compuestos activos como calcio en matrices alimentarias, como la mora de Castilla.

Conflicto de intereses: El manuscrito fue preparado y revisado con la participación de todos los autores, quienes declaramos que no existe conflicto de intereses que ponga en riesgo la validez de los resultados presentados. 
Tabla 3. Optimización experimental de mora de Castilla con RC+Ca.

\begin{tabular}{|c|c|c|c|c|c|c|}
\hline Variable & Objetivo & Peso & Impacto & $\begin{array}{c}\text { Óptimo } \\
\text { teórico }\end{array}$ & $\begin{array}{c}\text { Valor } \\
\text { experimental }\end{array}$ & $\begin{array}{c}\text { Error } \\
\text { residual (\%) }\end{array}$ \\
\hline $\mathrm{pH}$ & Maximizar & 0,6 & 3 & 2,76 & $2,69 \pm 0,01$ & 2,5 \\
\hline Acidez & Minimizar & 0,6 & 3 & 2,71 & $2,62 \pm 0,08$ & 3,3 \\
\hline${ }^{\circ}$ Brix & Maximizar & 0,6 & 3 & 8,80 & $8,07 \pm 0,15$ & 8,3 \\
\hline $\mathrm{Xw}$ & Minimizar & 0,6 & 3 & 83,64 & $83,7 \pm 1,7$ & 0.0 \\
\hline $\mathrm{aw}_{\mathrm{w}}$ & Minimizar & 0,6 & 3 & 0,95 & $0,964 \pm 0,01$ & 1,5 \\
\hline $\mathrm{Fr}$ & Maximizar & 1,0 & 5 & 12,22 & $12,35 \pm 0,74$ & 1,0 \\
\hline $\mathrm{FT}$ & Maximizar & 0,6 & 3 & 93,77 & $105,32 \pm 4,55$ & 12,3 \\
\hline $\mathrm{DPPH}$ & Maximizar & 0,6 & 3 & 336,97 & $319,26 \pm 9,45$ & 5,2 \\
\hline $\mathrm{ABTS}$ & Maximizar & 0,6 & 3 & 825,08 & $962,73 \pm 15,08$ & 16,7 \\
\hline $\mathrm{L} *$ & Minimizar & 0,6 & 3 & 18,30 & $22,51 \pm 0,71$ & 23,0 \\
\hline $\mathrm{a}^{*}$ & Maximizar & 0,6 & 3 & 26,53 & $8,47 \pm 0,88$ & 68,0 \\
\hline $\mathrm{b} *$ & Minimizar & 0,6 & 3 & 13,89 & $2,12 \pm 0,37$ & 84,7 \\
\hline Ca & Maximizar & ---- & ---- & ---- & $47,03 \mathrm{mg}$ Calcio/100g & -- \\
\hline
\end{tabular}

\section{REFERENCIAS}

1. ACEVEDO, F.; SOLIVA, R. 2017. Nanoemulsions as edible coatings. Curr. Opin. Food Sci. (Netherlands). 15:43-49. https://doi.org/10.1016/j.cofs.2017.06.002

2. ALI, A.; MAQBOOL, M.; RAMACHANDRAN, S.; ALDERSON, P. 2012. Gum arabic as a novel edible coating for enhancing shelf-life and improving postharvest quality of tomato (Solanum lycopersicum L.) fruit. Postharvest Biol Technol. (Netherlands). 58:42-47. https://doi. org/10.1016/j.postharvbio.2010.05.005

3. AOAC. 2012. Official methods of analysis. Association of Official Analytical Chemists. Arlington. 19th edition. Arlington, Virginia (USA). 684p.

4. ASOCIACIÓN PROVINCIAL DE APICULTORES DE GRANADA-APAG. 2016. Descontaminación industrial de ceras. Valorización de la cera y sus subproductos. Informe final de investigación. (España). p.26. Disponible desde Internet en: http://apinevada.com/media/files/news/ Memoriafinal1.pdf (con acceso el 18/01/2018).

5. CARVAlHO, C.; BETANCOUR, A. 2015. Quality characterization of Andean blackberry fruits (Rubus glaucus Benth.) in different maturity stages in Antioquia. Agron Colomb. (Colombia). 33(1):74-83. https://doi. org/10.15446/agron.colomb.v33n1.47132

6. CHIEN, P.; SHEU, F.; YANG, F. 2007. Effects of edible chitosan coating on quality and shelf life of sliced mango fruit. J. Food. Eng. (England). 78(1):225-229. https://doi. org/10.1016/j.jfoodeng.2005.09.022
7. FALGUERA, V.; QUINTERO, J.; JIMÉNEZ, A.; MUÑOZ, J.; IBARZ, A. 2011. Edible films and coatings: Structures, active functions and trends in their use. Trends Food Sci Technol. (England). 22(6): 292-303. https://doi. org/10.1016/j.tifs.2011.02.004

8. FRANCESCHINIS, L.; SALVATORI, D.M.; SOSA, N.; SCHEBOR, C. 2014. Physical and Functional Properties of Blackberry Freeze- and Spray-Dried Powders. Dry Techno. (USA). 32(2):197-207. https://doi.org/10.1080/ 07373937.2013 .814664

9. GALUS, S.; KADZIŃSKA, J. 2015. Food applications of emulsion-based edible films and coatings. Trends Food Sci. Technol. (England). 45(2):273-283. https://doi. org/10.1016/j.tifs.2015.07.011

10. GARCÍA, M.; DE LA PAZ, N.; FERNÁNDEZ, M.; VALDÉZ, J. 2014. Evaluación de la actividad antioxidante de disoluciones de sales de quitosana. Ciencia y Tecnología de Alimentos. (Cuba). 24(3):62-66.

11. GARZÓN, G.; RIEDL, K.; SCHWARTZ, S. 2009. Determination of anthocyanins, total phenolic content, and antioxidant activity in Andes berry (Rubus glaucus Benth). J. Food Sc. (USA). 74(3):227-232. https://doi. org $/ 10.1111 /$ j.1750-3841.2009.01092.x

12. GHASEMNEZHAD, M.; NEZHAD, M.; SOMAYEH, M. 2010. Changes in Postharvest Quality of Loquat (Eriobotrya japonica) Fruits Influenced by Chitosan. Hort. Environ. Biotechnol. (Korean). 52(1):40-45. https://doi. org/10.1007/s13580-011-0028-5 
13. GRIJALBA, C.; CALDERÓN, L.; PÉREZ, M. 2010. Rendimiento y calidad de la fruta en mora de castilla (Rubus glaucus), con y sin espinas, cultivada en campo abierto en Cajicá (Cundinamarca, Colombia). Rev. Fac. Cienc. Básicas. (Colombia). 6(1):24-41.

14. HERNÁNDEZ-MUÑOZ, P.; ALMENAR, E.; VALLE, V.D.; VELEZ, D.; GAVARA, R. 2008. Effect of chitosan coating combined with postharvest calcium treatment on strawberry (Fragaria $\mathrm{x}$ ananassa) quality during refrigerated storage. Food Chem. (England). 110(2):428-435. https:// doi.org/10.1016/j.foodchem.2008.02.020

15. INSTITUTO COLOMBIANO DE NORMAS TÉCNICAS Y CERTIFICACIÓN NORMA-ICONTEC. 1997. Norma técnica colombiana 4106. Frutas frescas. Mora de castilla. Especificaciones. Bogotá, Colombia. 13p.

16. KERCH, G.; SABOVICS, M.; KRUMA, Z. 2011. Effect of chitosan and chitooligosaccharide on vitamin $\mathrm{C}$ and polyphenols contents in cherries and strawberries during refrigerated storage. Eur. Food Res. Technol. (Germany). 233:351-358. https://doi.org/10.1007/s00217-011-1525-6

17. KERCH, G. 2015. Chitosan films and coatings prevent losses of fresh fruit nutritional quality: A review. Trends Food Sci. Technol. (England). 46(2):159-166. https://doi. org/10.1016/j.tifs.2015.10.010

18. KUMARI, M.; MAHAJAN, H.; JOSHI, R.; GUPTA, M. 2017. Development and structural characterization of edible films for improving fruit quality. Food Packaging and Shelf Life. (Alemania). 12:42-50.

19. LÓPEZ-MATA, M.; RUIZ-CRUZ, S.; NAVARROPRECIADO, C. 2012. Effect of chitosan edible coatings in the microbial reduction and conservation of the quality of strawberries. Biotecnia. (México). 14(1):33-43.

20. MA, L.; ZHANG, M.; BHANDARI, B.; GAO, Z. 2017. Recent developments in novel shelf life extension technologies of fresh-cut fruits and vegetables. Trends Food Sci. Technol.. (Netherlands). 64:23-38. https://doi.org/10.1016/ j.tifs.2017.03.005

21. MANNOZZI, C.; CECCHINI, J,P.; TYLEWICSZ, U.; SIROLI, L. 2017. Study on the effi cacy of edible coatings on quality of blueberry fruits during shelf-life. LWT - Food Sci. Technol. (USA). 85:440-444. https://doi.org/10.1016/j. lwt.2016.12.056

22. MANTILLA, N.; CASTELL-PEREZ, M.E.; GOMES, C.; MOREIRA, R.G. 2012. Multilayered antimicrobial edible coating and its effect on quality and shelf-life of fresh-cut pineapple (Ananas comosus). LWT - Food Sci.
Technol. (USA). 51(1):37-43. https://doi.org/10.1016/ j.lwt.2012.10.010

23. MORENO, C.; ANDRADE, M.; TERÁN, A. 2016. Efecto del uso combinado de radiación uv-c y atmósfera modificada sobre el tiempo de vida útil de mora de Castilla. Rev Iberoam Tecnol Postcosecha. (México). 17(1):71-78.

24. OLIVEIRA, D.M.; KWIATKOWSKI, A.; ROSA, C.I.L.F.; CLEMENTE, E. 2014. Refrigeration and edible coatings in blackberry (Rubus spp.) conservation. J. Food Sci. Technol. (India). 51(9):2120-2126. https://doi.org/10.1007/s13197012-0702-3

25. OSZMIAŃSKI, J.; NOWICKA, P.; TELESZKO, M.; WOJDYŁO, A.; CEBULAK, T.; OKLEJEWICZ, K. 2015. Analysis of phenolic compounds and antioxidant activity in wild blackberry fruits. Int. J. Mol. Sci. (Switzerland). 16:14540-14553. https://doi.org/10.3390/ijms160714540

26. PÉREZ-GALLARDO, A.; GARCÍA-ALMENDÁREZ, B.; BARBOSA-CÁNOVAS, G. 2014. Effect of starch-beeswax coatings on quality parameters of blackberries (Rubus spp.). J. Food Sci. Technol. (India). 52(9): 5601-5610. https://doi. org/10.1007/s13197-014-1665-3

27. PETRICCIONE, M.; PASQUARIELLO, M.; MASTROBUONI, F.; ZAMPELLA, L.; DI PATRE, D. 2015. Influence of a chitosan coating on the quality and nutraceutical traits of loquat fruit during postharvest life. Sci. Hortic. (Netherlands). 197:287-296. https://doi. org/10.1016/j.scienta.2015.09.051

28. RAMÍREZ, J.; ARISTIZÁBAL I.; RESTREPO, J. 2013. Conservación de mora de Castilla mediante la aplicación de un recubrimiento comestible de gel de mucílago de penca de sábila. Vitae. (Colombia). 20(3):172-183.

29. RAYBAUDI-MASSILIA, M.; MOSQUEDA-MELGAR, J.; MARTÍN-BELLOSO, O. 2008. Edible alginate-based coating as carrier of antimicrobials to improve shelf-life and safety of fresh-cut melon. Int. J Food Microbiol. (Netherlands) 121(3):313-327. https://doi.org/10.1016/ j.ijfoodmicro.2007.11.010

30. ROJAS-GRAÜ, A.; AVENA-BUSTILLOS, J.; OLSEN, C.; FRIEDMAN, M.; HENIKA, P.R.; MARTÍNBELLOSO, O. 2007. Effects of plant essential oils and oil compounds on mechanical, barrier and antimicrobial properties of alginate-apple puree edible films. J. Food Eng. (England). 81(3):634-641. https://doi.org/10.1016/ j.jfoodeng.2007.01.007

31. RODRÍGUEZ, L.; LÓPEZ, L.; GARCÍA, M. 2010. Determinación de la composición química y actividad 
antioxidante en distintos estados de madurez de frutas de consumo habitual en Colombia, Mora (Rubus glaucus B.), Maracuyá (Passiffora edulis), Guayaba (Psidium guajava L.) Y Papayuela (Carica cundinam). Revista de Alimentos. (Colombia) 19(21):16-34.

32. RODRÍGUEZ, B.; ZULUAGA-PAVA, Y.; CRUZ-RIOS, D. 2012. Producto potencialmente simbiótico a partir de mora de castilla (Rubus glaucus) aplicando impregnación a vacío. Scientia Agropecuaria. (Colombia) 3:273-278.

33. TOSNE, Z.; MOSQUERA, S.; VILLADA, H. 2014. Efecto de recubrimiento de almidón de yuca y cera de abejas sobre el chontaduro. Biotecn. Sector Agropec. y Agroindustr. (Colombia). 12 (2):30-39.
34. VELICKOVA, E.; WINKELHAUSEN, E.; KUZMANOVA, S.; ALVES, V.; MOLDÃO-MARTINS, M. 2013. Impact of chitosan-beeswax edible coatings on the quality of fresh strawberries (Fragaria ananassa cv camarosa) under commercial storage conditions. LWT - Food Science and Technology. (England). 52(2):80-92. https://doi. org/10.1016/j.lwt.2013.02.004

35. VILLEGAS, C.; ALBARRACÍN, W. 2016. Conservación de mora de Castilla (Rubus glaucus Benth) mediante la aplicación de un recubrimiento comestible. Vitae. 23(1):727-730.

36. ZHANG, W.; XIAO, A.; QIANBA, L. 2014. Beeswax-chitosan emulsion coated paper with enhanced water vapor barrier efficiency. Appl. Surf. Sci. (Netherlands) 300:80-88. https:// doi.org/10.1016/j.apsusc.2014.02.005 